\title{
Scattering contribution to the double-pass PSF using Monte Carlo simulations
}

\author{
Dimitrios Christaras ${ }^{1,2 *}$, Harilaos Ginis ${ }^{3,1}$, Alexandros Pennos ${ }^{1}$ and Pablo Artal ${ }^{1}$ \\ ${ }^{1}$ Laboratorio de Óptica, Universidad de Murcia, Murcia, Spain \\ ${ }^{2}$ UCL Institute of Ophthalmology, University College London, London, England \\ ${ }^{3}$ Department of Research, Athens Eye Hospital, Athens, Greece \\ *Corresponding author: d.christaras@ucl.ac.uk
}

\begin{abstract}
Purpose: Scattering in the eye occurs mainly at two sites: the eye's optical media and the deeper retinal layers. Although the two phenomena are often treated collectively, their spatial domain of contribution to the double-pass Point Spread Function (PSF) is different: the fundus effect is limited to the narrow and middle part of the PSF whereas scattering in the eye's optics extends also to wide angles. The objective of this work was to determine the domain of contribution at the double-pass PSF of light scattered in the ocular media and the ocular fundus, using simulated and experimental data for two different wavelengths and for two different pigmentations.

Methods: Diffuse reflection was simulated using Monte Carlo simulations for a 4-layer retinal fundus model. Four situations were simulated in total for two different choroidal absorptions at two different wavelengths. Light diffusion in the fundus was the only phenomenon considered in the model. The simulations were compared against experimental fundus reflection data obtained in a previous study.

Results: The simulations showed that at $560 \mathrm{~nm}$, diffusion in the fundus causes light to extend to a radius of 2 degrees, independently of the choroidal pigmentation, whereas at $650 \mathrm{~nm}$ it extends to radii of 4.5 degrees and 4 degrees for low and high choroidal pigmentation respectively. Experimental data showed a similar behaviour at low angles where light diffusion in the fundus is dominant, but different at higher angles due to scattering in the ocular media.

Conclusion: The spatial contribution of light diffused in the ocular fundus to the PSF was found to be limited to narrower angles compared to that of scattering at the ocular media. The comparison of simulated and optical data showed that beyond 2 degrees at $560 \mathrm{~nm}$ and 4 to 4.5 degrees at $650 \mathrm{~nm}$ the only phenomenon contributing to the PSF is scattering in the ocular media, whereas the fundus contribution can be assumed as negligible.
\end{abstract}

Keywords: Straylight; Ocular Scattering; Fundus Reflectometry

\section{Introduction}

In double-pass ophthalmic systems $(1,2)$ that involve shining structured light to the retina $(3,4)$, the captured light is affected by a number of phenomena: diffraction at the pupil, aberrations and scattering in the ocular fundus and the eye's optical media. Diffraction and aberrations affect solely high frequencies whereas scattering extents to lower frequencies as well. Scattering in the fundus, also called fundus diffusion, and scattering in the ocular media are often treated as a single phenomenon, although their spatial domains of dominance can be significantly different, with fundus diffusion being generally limited to narrower angles than that of the optical media.

The amount of scattering introduced by the fundus depends on the light's wavelength: shorter wavelengths are strongly absorbed whereas longer wavelengths scatter and propagate deeper into the fundus. This wavelength dependence is attributed primarily to two absorbers: melanin and haemoglobin. Melanin exhibits its peak absorption at about $335 \mathrm{~nm}$ and falls rapidly with increasing wavelength, absorbing several orders of magnitude less at wavelengths over $700 \mathrm{~nm}$ (5). Haemoglobin exhibits an absorption spectrum that depends on the concentration of oxygen but generally, has a global maximum at about $400 \mathrm{~nm}$, a local maximum at about $530 \mathrm{~nm}$ and it decreases significantly over $700 \mathrm{~nm}(6)$. At the fovea, an additional pigment 
exists, the macular pigment, which absorbs the lower end of the visible spectrum, possibly acting as a protective filter against photo-oxidation by blue light (7).

Melanin can be found in the choroid and in the Retinal Pigment Epithelium (RPE) with the choroidal melanin being highly variable amongst the population and related to the iris pigmentation, contrary to the RPE melanin which appears to be constant (8). Haemoglobin is present in the vascular choroid and is considered constant amongst the healthy population. Since choroidal melanin is the only variable absorber in the healthy population, diffuse fundus reflection is expected to be higher for individuals with lower melanin pigmentation than those with higher melanin pigmentation. Moreover, the choroid, the iris and the ciliary body are part of the same anatomical layer, the uvea, and consequently, there is a strong correlation between choroidal melanin pigmentation and the pigmentation of the iris (9).

Scattering in the ocular media, for the healthy population, depends generally on age, being proportional to the fourth power of the individual's age (10). Moreover, it has been shown that scattering has a weak wavelength dependence, with medium wavelengths showing the lowest amounts of scattering (11).

The main tool used in this study is the Monte Carlo simulation. It has been extensively used for the study of light propagation in human tissue $(12,13)$ and specifically to model light propagation in the ocular fundus (14-16). Monte Carlo simulations were used to establish the spatial contribution of light diffusion in the fundus to the PSF. For this purpose, a fundus model was considered and diffuse light in the fundus at two different wavelengths and for two different choroidal absorptions was simulated. The simulated data were, subsequently, compared against experimental data from fundus reflectance values collected from two different melanin groups at the above wavelengths from a previous study (17).

The objective of the study was the analysis of the spatial characteristics of the reflected fundus light. More specifically, the study aimed to determine, using simulations, the spatial domain of contribution for fundus diffusion and compare it to that of scattering in the optical media observed in experimental fundus reflection data.

The study, apart from exploring the spatial and spectral characteristics of the reflected light, can also act as a guide in the design of setups where the spatial components of the PSF are important.

\section{Methods}

A simplified 4-layer model of the retinal fundus was considered, where light can be absorbed, scattered or reflected at the interface between the different layers. The model consisted of the photoreceptor layer, the Retinal Pigment Epithelium (RPE), the choroid, the sclera and two additional non-absorbing, non-scattering layers, one posterior and one anterior, with the refractive indexes of tissue and vitreous, respectively. For each layer absorption and scattering coefficients $\mu_{\mathrm{a}}$ and $\mu_{\mathrm{s}}$ were defined, as well as a thickness and a refractive index for each layer. Finally, an additional parameter $g$ was introduced to describe anisotropy of scattering in the media. The simulations were done using a $C$ program developed by Wang and Jacques (18) that simulates light propagation in multi-layered tissue using the Monte Carlo method. The values for the coefficients used were based on the work done by Hammer et al. (14), where absorption and scattering of the ocular fundus were studied through the ex-vivo measurement of the transmittance and the reflectance of isolated fundus layers, and using the inverse Monte Carlo simulation. The result from the simulation was the intensity of the back-scattered light at the surface of the retina, i.e. the PSF.

Four simulations were carried out in total, resulting in four corresponding PSFs. The four simulations aimed to describe back-scattered light due to diffusion in the fundus at two different wavelengths at $560 \mathrm{~nm}$ and $650 \mathrm{~nm}$ with two different amounts of choroidal melanin for each wavelength. To study the effect of choroidal melanin changes seen in the population, the absorption coefficient of the choroid was decreased by about two thirds (65\%) for the above wavelengths. Although this may seem arbitrary, choroidal melanin concentrations exhibit such variability between individuals (19). The parameters used in the simulations can be found in the appendix.

A first estimate on the relative contribution of fundus diffusion and scattering at the ocular media was done through the comparison of the simulated PSFs with the PSF for the standard observer (20), which is an estimate of the wide-angle PSF, affected by both fundus and optical media scattering, for the standard 
observer defined by Commission Internationale d'Eclairage (CIE). This PSF was based on psychophysical and physiological data and depends heavily on age; however, it does not include wavelength dependence.

Subsequently, each PSF was convolved with a set of binary disks of increasing radius and the intensity at the centre of the resulting disk was extracted. Each simulated disk was the equivalent of a projected disk of the corresponding radius on the retina. Comparing intensities at the centre of a disk of a specific radius (in degrees of visual angle) is an indirect way of comparing PSFs at the respective angle since the intensity at the centre of the disk and the PSF are related through integration and consequently the PSF at a given angle can be computed by differentiating the intensity at the centre of a disk of the corresponding size (3). There are two advantages of comparing intensity values instead of PSFs. Firstly, differences that might not be visible at a PSF level are emphasized through the integration. Secondly, the signal to noise ratio for the same spatial point is higher, which is particularly important at large angles where the PSF drops significantly.

The simulated intensities were compared against optical data retrieved in a previous study for a set of six subjects using a double-pass system. The system and the respective method followed for the extraction of data have been thoroughly described (17).

Finally, the simulated intensity curves were normalized to each curve's maximum value, and the spatial values where the derivative became zero were extracted. These values denoted the range of contribution of light diffusion in the fundus. Any contribution, then observed in the experimental PSF outside this value can be exclusively attributed to scattering at the ocular media.

\section{Results}

Figure 1 shows the PSF for fundus diffusion at $560 \mathrm{~nm}$ (green) and $650 \mathrm{~nm}$ (red) as well as the complete PSF for the standard observer for an age of 40 years (black). Since the PSF for the standard observer includes scattering in the ocular media and fundus diffusion it is expected to be higher at larger angles than the simulated PSFs that describe only fundus diffusion phenomena. At $560 \mathrm{~nm}$ the simulated PSF drops significantly outside 2 degrees, whereas at $650 \mathrm{~nm}$ the PSF is still high due to the increased light diffusion in the fundus.

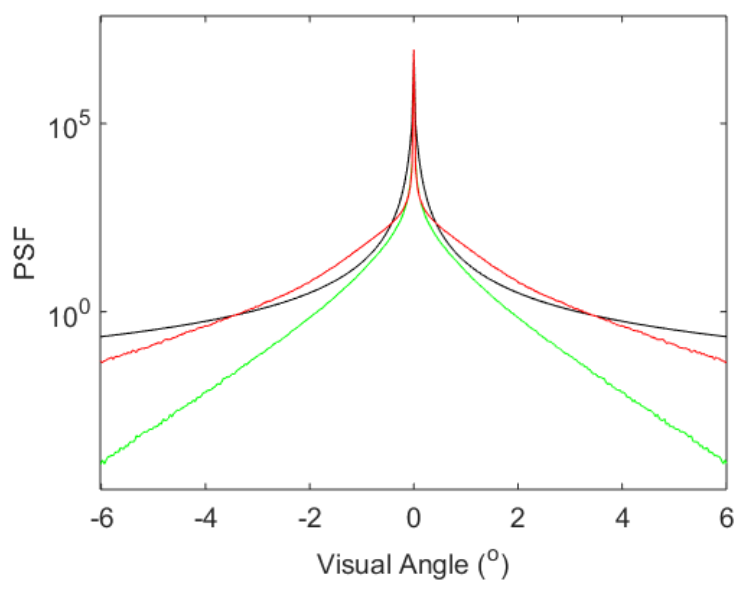

Figure 1. Simulated PSFs for a high pigmentation fundus at 560nm (green), 650nm (red) and the PSF CIE for the standard observer (black).

Subsequently, the simulated PSFs were convolved with a set of 21 binary disks of increasing radius, from 0.17 to 7 degrees of visual angle, and the intensity at the centre of each disk was extracted. Figure 2(a) shows the intensity at the centre of the simulated disks plotted against their radius, for the four different cases: low pigmentation and high pigmentation at $560 \mathrm{~nm}$ and at $650 \mathrm{~nm}$. The simulated data are depicted in dashed 
lines whereas the experimental data collected in a previous study (17) are depicted in filled circles with the error bars denoting standard deviations.

The PSF and the intensity at the centre are related through differentiation and therefore when the intensity curve becomes flat, the PSF at that angle will be 0 . Since the simulated data describe solely diffuse light in the fundus, their contribution to the PSF would vanish when the intensity curves reach a plateau.

In the simulated curves, an increase in the disk's radius would mean more light available at its centre originating from the outskirts of the disk. This light depends heavily on the absorption and scattering of the fundus: when absorption is high, little light from the outskirts can reach the disk's centre. There will be, therefore, a specific disk radius where light from the outskirts no longer reaches the centre, causing the early plateau shown in the bottom curves in figure 2(a). When absorption is low, as is the case at $650 \mathrm{~nm}$, the plateau is observed at larger disk radii, seen in the top two simulated curves in figure 2(a). The increase in the reflectance for the simulated curves for the two different pigmentations was $2.6 \%$ at $560 \mathrm{~nm}$ and $30.5 \%$ at $650 \mathrm{~nm}$, showing, as expected, that choroidal melanin density is more significant at longer wavelengths.

Similar behaviour can be observed in the experimental data, for the two subject groups: the mean observed increase for the low and high pigmentation groups was $1.5 \%$ and $37.9 \%$ at $560 \mathrm{~nm}$ and $650 \mathrm{~nm}$ respectively. The experimental data, however, apart from contribution of the fundus, were largely affected by scattering in the ocular media. This can be observed in Fig. 2(a): in the simulated curves the derivative becomes zero at a lower angle than that of the corresponding experimental data. The difference in the slopes between the experimental and simulated intensities can be attributed exclusively to scattering at the ocular media, present solely in the experimental data.

A better way to visualize the plateaus in the intensity curves is to normalize them with the highest intensity value of each curve, as seen in Fig.2(b). The plateaus at the two $560 \mathrm{~nm}$ curves start at 2 degrees, whereas at $650 \mathrm{~nm}$ they start at 4 degrees and 4.5 degrees for high and low melanin concentrations respectively.

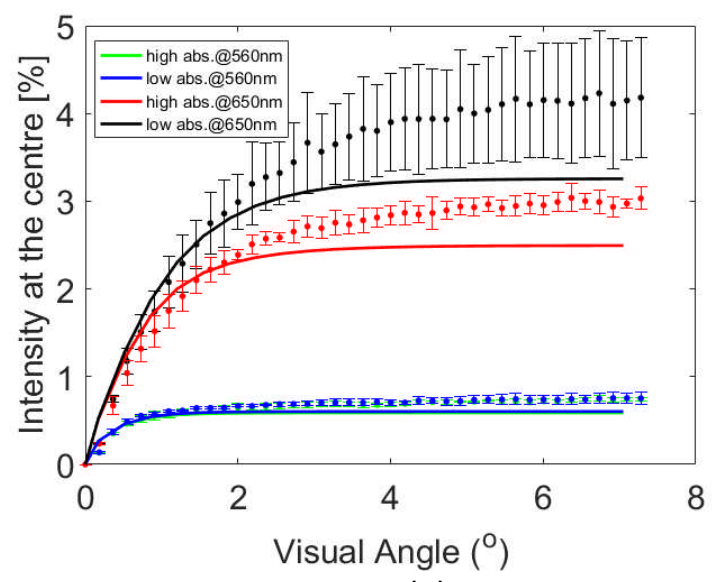

(a)

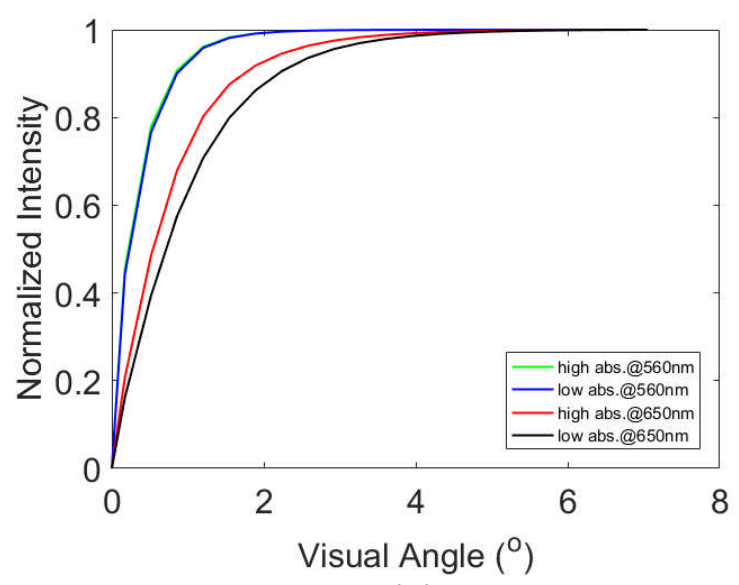

(b)

Figure 2. (a) Simulated and experimental intensities and (b) normalized simulated intensities at the centre of disks with increasing radius, for two wavelengths and two different choroidal pigmentations.

\section{Discussion}

A Monte Carlo fundus model was built to describe diffuse light in the fundus at two different wavelengths and two different choroidal pigmentation levels. The objective was to effectively simulate fundus reflectance values when only light diffusion is considered and find its spatial domain of contribution. The simulated data were then compared against experimental data acquired from a double pass setup for two different groups classified according to their iris pigmentation, which is known to be in direct relation to the pigmentation of the choroid. It was shown that light diffusion in the fundus depends on the wavelength of the incident light, 
with longer wavelengths being diffused more and, consequently causing a higher increase to the outskirts of the PSF. Fundus diffusion contribution at $650 \mathrm{~nm}$ stopped at 4 degrees for high pigmented individuals and 4.5 degrees for low pigmented ones. At $560 \mathrm{~nm}$, on the other hand, pigmentation had no effect, where both pigmentation scenarios showed a contribution limited to up to 2 degrees. For wider angles, the only contribution to the PSF is that of scattering at the ocular media, seen in the experimental data.

The wavelengths used in the simulation were chosen to be at the middle and long part of the visible spectrum and also match the wavelengths used in the optical data from the previous study.

It needs to be noted that the simulation needed a much higher sampling for narrow angles compared to that of wider angles. This is because the PSF at narrow angles exhibits a fast decrease with angle as seen in figure 2 that can be as high as 1000 times for a third of a degree. For this reason, the peak of the PSF was calculated in a separate simulation that focused exclusively on the narrow part of the PSF.

The simulated reflectances had to be scaled to be in agreement with the experimental reflectances. The scale factor was the same for all 4 simulated intensity curves and was determined by matching the narrow angle part of the $560 \mathrm{~nm}$ experimental and simulated curves. The need for scaling raises from the fact that, in the model, the simulated fundus images were taken to be a binary. Real fundus images however, contain different structures and also exhibit specular reflections which would affect the absolute intensity values at the centre of the disks considerably. The absolute values, however, are of no interest; the information on the spatial contribution can be extracted solely by relative data.

\section{Conclusion}

The qualitative effect of scattering in the ocular media and light diffusion in the deeper fundus layers on the PSF is similar; both phenomena cause the symmetric scatter of the light and a consequent increase to the outskirts of the PSF. Quantitatively, however, the two phenomena can be very distinct; the fundus shows strong wavelength dependence, with red light extending to 4 and 4.5 degrees radius for high and low pigmented individuals, respectively, and with green light extending to 2 degrees radius independently of the pigmentation. The optical media, on the other hand, show weaker wavelength dependence and wider spatial distribution. The above observation can be useful in optical systems where the spatial distribution of structured light is important.

\section{Disclosure}

The authors report no conflicts of interest and have no proprietary interest in any of the materials mentioned in this article.

\section{Acknowledgements}

This work was supported by the European Research Council Advanced Grant ERC-2013-AdG-339228 (SEECAT), the Spanish SEIDI, grant FIS2013-41237-R and the European Commission PITN-GA-2010-264605 (ITN OpAL).

\section{References}

1. Campbell FW, Gubisch RW. Optical quality of the human eye. J Physiol. 1966;186(3):558-78.

2. Santamaría J, Artal $P$, Bescós J. Determination of the point-spread function of human eyes using a hybrid optical-digital method. J Opt Soc Am A. 1987;4(6):1109-14.

3. Ginis H, Pérez GM, Bueno JM, Artal P. The wide-angle point spread function of the human eye reconstructed by a new optical method. J Vis. 2012;12(3):1-10.

4. Lochocki B, Gambín A, Manzanera S, Irles E, Tajahuerce E, Lancis J, Artal P. Single pixel camera ophthalmoscope. Optica. 2016;3(10):1056. 
5. Anderson RR, Parrish JA. The optics of human skin. J Invest Dermatol. 1981;77(1):13-9.

6. Horecker BL. The absorption spectra of Hemoglobin and its derivatives in the visible and near infrared regions. J Biol Chem. 1943;148:173-83.

7. Kirschfeld K. Carotenoid pigments: their possible role in protecting against photooxidation in eyes and photoreceptor cells. Proc R Soc London. 1982;216(1202):71-85.

8. Weiter JJ, Delori FC, Wing GL, Fitch KA. Retinal pigment epithelial lipofuscin and melanin and choroidal melanin in human eyes. Investig Ophthalmol Vis Sci. 1986;27(2):145-52.

9. Delori FC, Pflibsen KP. Spectral reflectance of the human ocular fundus. Appl Opt. 1989;28(6):106177.

10. Vos JJ, Van den Berg TJTP. On the course of the disability glare function and its attribution to components of ocular scatter. CIE Collect Colour Vis. 1997;124(2):11-29.

11. Ginis H, Perez GM, Bueno JM, Pennos A, Artal P. Wavelength dependence of the ocular straylight. Invest Ophthalmol Vis Sci. 2013;54(5):3702-8.

12. Simpson CR, Kohl M, Essenpreis M, Cope M. Near-infrared optical properties of ex vivo human skin and subcutaneous tissues measured using the Monte Carlo inversion technique. Phys Med Biol. 1998;43(9):2465-78.

13. Patwardhan S V, Dhawan AP, Relue PA. Monte Carlo simulation of light-tissue interaction: threedimensional simulation for trans-illumination-based imaging of skin lesions. IEEE Trans Biomed Eng. 2005;52(7):1227-36.

14. Hammer M, Roggan A, Schweitzer D, Müller G. Optical properties of ocular fundus tissues--an in vitro study using the double-integrating-sphere technique and inverse Monte Carlo simulation. Phys Med Biol. 1995;40(6):963-78.

15. Guo Y, Yao G, Lei B, Tan J. Monte Carlo model for studying the effects of melanin concentrations on retina light absorption. J Opt Soc Am A Opt Image Sci Vis. 2008;25(2):304-11.

16. Preece SJ, Claridge E. Monte Carlo modelling of the spectral reflectance of the human eye. Phys Med Biol Phys Med Biol. 2002 Aug 21;47(47):2863-77.

17. Christaras D, Ginis H, Artal P. Spatial properties of fundus reflectance and red-green relative spectral sensitivity. J Opt Soc Am A. 2015;32(9):1723-8.

18. Wang L, Jacques SL. Monte Carlo modeling of light transport in multi-layered tissues in standard C. University of Texas M. D. Anderson Cancer Center; 1992.

19. Wakamatsu K, Hu D-NN, Mccormick SA, Ito S. Characterization of melanin in human iridal and choroidal melanocytes from eyes with various colored irides. Pigment Cell Melanoma Res. 2008;21(1):97-105.

20. Vos JJ, Van den Berg TJTP. Report on disability glare. CIE Collect Colour Vis. 1999;135.

\section{Appendix}

The parameters used in the simulations are shown in the table below. In order to consider Fresnel reflection at the first and last interface two non-scattering and non-absorbing layers was considered to simulate vitreous humor and tissue before the photoreceptors and after the sclera respectively. In order to simulate population with lighter choroidal/iridal pigmentation the absorption coefficient was reduced by about $65 \%$.

Table 1. Optical parameters for the different layers of the Monte Carlo model for the two wavelengths and two different choroidal densities, where $\mu_{\mathrm{a}}$ and $\mu_{\mathrm{s}}$ are the absorption and scattering coefficients respectively in $\mathrm{cm}^{-1}, \mathrm{~g}$ is the anisotropy coefficient and $\mathrm{d}$ is the thickness of the layer in $\mathrm{cm}$. 


\begin{tabular}{|c|c|c|c|c|c|}
\hline Wavelength [nm] & Layer & $\boldsymbol{\mu}_{\mathrm{a}}\left[\mathbf{c m}^{-\mathbf{1}}\right]$ & $\boldsymbol{\mu}_{\mathrm{s}}\left[\mathbf{c m}^{-\mathbf{1}}\right]$ & $\mathbf{g}$ & $\mathbf{d}[\mathbf{c m}]$ \\
\hline \multirow{5}{*}{560} & $\mathrm{Ph}$ & 45 & 310 & 0.97 & 0.02 \\
\cline { 2 - 6 } & $\mathrm{RPE}$ & 1000 & 1200 & 0.84 & 0.001 \\
\cline { 2 - 6 } & Choroid & 230 & 800 & 0.94 & 0.025 \\
\cline { 2 - 6 } & Sclera & 4 & 910 & 0.9 & 0.07 \\
\hline \multirow{5}{*}{650} & Ph & 15 & 250 & 0.97 & 0.02 \\
\cline { 2 - 6 } & RPE & 530 & 1200 & 0.84 & 0.001 \\
\cline { 2 - 6 } & Choroid & 50 & 500 & 0.94 & 0.025 \\
\cline { 2 - 6 } & Sclera & 2 & 750 & 0.9 & 0.07 \\
\hline \multirow{5}{*}{560} & Ph & 45 & 310 & 0.97 & 0.02 \\
\cline { 2 - 6 } & RPE & 1000 & 1200 & 0.84 & 0.001 \\
\cline { 2 - 6 } & Choroid & 80 & 800 & 0.94 & 0.025 \\
\cline { 2 - 6 } & Sclera & 4 & 910 & 0.9 & 0.07 \\
\hline \multirow{6}{*}{650} & Ph & 15 & 250 & 0.97 & 0.02 \\
\cline { 2 - 6 } & RPE & 530 & 1200 & 0.84 & 0.001 \\
\cline { 2 - 6 } & Choroid & 17 & 500 & 0.94 & 0.025 \\
\cline { 2 - 6 } & Sclera & 2 & 750 & 0.9 & 0.07 \\
\hline
\end{tabular}

